\title{
CULTURA, IDENTIDADE E TRABALHO: INTER-RELAÇÃO DE CONCEITOS
}

\author{
CULTURE, IDENTITY AND WORK: INTERRELATION OF CONCEPTS \\ CULTURA, IDENTIDAD Y TRABAJO: INTERRELACIÓN DE CONCEPTOS
}

\author{
Laura Filomena Santos de Araújo Netto ' \\ Flávia Regina Souza Ramos ${ }^{2}$
}

\begin{abstract}
RESUMO: O artigo pretende uma reflexão em torno da inter-relação dos conceitos de cultura identidade e trabalho, entendendo-a como contribuiçăo aos estudos que buscam compreender os processos de construção da identidade do enfermeiro. Parte do pressuposto que essa identidade se constrói no cotidiano de trabalho e, dentre outras referências, numa dada cultura.
\end{abstract}

PALAVRAS-CHAVE: cultura, identidade, trabalho, Enfermagem

As categorias centrais de um estudo são consoantes à natureza do objeto a ser desvelado, fundam ou permitem uma apropriação do mesmo num determinado contexto teórico de abordagem.

Em nosso estudo elegemos cultura, identidade e trabalho, a partir dos quais acreditamos estabelecer delineamentos iniciais para a proposição de uma base conceitual para a análise do processo de construção de identidades profissionais, no caso, do profissional enfermeiro, onde cultura vem afirmar a relação dialética homem/homem/mundo em sua realidade subjetiva/objetiva e busca trazer o aspecto conflitivo das relações dos homens e o seu potencial transformador; identidade é o conceito articulador necessário; e trabalho é o locus de produção do homem e onde se dão as suas escolhas. Na perspectiva particular deste estudo procuramos uma re-apropriação e inter-relação desses conceitos.

Parte-se do pressuposto de que estudos em perspectiva de cultura, identidade e trabalho podem ajudar a compreender as relações significantes do ser/fazer do enfermeiro, no âmbito do seu trabalho cotidiano.

\section{PORQUE FALAR EM CULTURA E IDENTIDADE}

Teorias de identidade se fundam na cultura e considera-se que cultura é apreendida, dinâmica e compartilhadamente; ambas estão imbricadas e ganham sentido no universo das relações sociais. Cultura e identidade são pertinentes, pois cultura não só é um produto da vivência dos homens, é também um processo dessa produção. E identidade é constituida sempre em uma certa relação com o onde o homem está situado: nessa relação o homem se define. As condições de existência do homem são aquilo através do qual ele se constitui. Então, o homem se dá sempre por perspectivas e ou delimitações.

\footnotetext{
'Enfermeira, docente do Depto. Enfermagem Médico Cirúrgica da Faculdade de Enfermagem e Nutrição da Universidade Federal de Mato Grosso (UFMT) e mestranda do Programa de Pós Graduação do Instituto de Saúde Coletiva - UFMT.

2 Doutora em Filosofia em Enfermagem, Docente do Departamento de Enfermagem da Universidade Federal de Santa Catarina.
} 


\section{COMO CONCEITUAR CULTURA AO REFERIR-SE SOBRE IDENTIDADE}

Observa-se que os conceitos de cultura são variados e divergentes em relação ao primado do mundo natural (habitat) ou social, material ou simbólico na formulação das idéias sobre as culturas humanas.

Deve-se observar as concepções ideológicas de cultura, comportamento cultural e natureza, enquanto construções da civilização ocidental, pois entende-se que esses variados conceitos foram constituidos historicamente pelas diversas sociedades, podendo servir-lhes como linguagens de poder/legitimação.

Considerando a complexidade de uma conceituação de cultura, pode-se apenas levantar aspectos importantes na construção desse conceito em relação ao referencial de identidade. Sendo assim, no conceito de cultura deve ser considerado que:

- na distinção entre os mundos - da natureza, homem e sociedade, tratamos da cultura como conceito que implica uma relação dialética entre esses mundos;

- que o homem é um ser de transformação e não em adaptação;

- tratamos dos fatores culturais, reportados aos valores e comportamentos sociais, enquanto produtos de contextos sociais e históricos;

- ser (a cultura) processo de produção do homem e sistema conceitual - aquele que permite aos homens a compreensão da realidade social.

\section{COMO SE DÁ A RELAÇÃO HOMEM/SOCIEDADE?}

Segundo Berger e Luckmann (1973), o homem é tanto um ser da natureza como da sociedade. Coexistem permanentemente animalidade e socialidade, numa relação dialética. Essa condição humana se manifesta em cada indivíduo.

A dialética apresenta-se entre o ser individual e o mundo sócio e historicamente estruturado, assim como entre o ser biológico e sua identidade socialmente produzida. A dialética manifesta-se, também, no funcionamento e na "limitação mútua do organismo e da sociedade" (Ibidem, p.237).

Sendo a relação natureza/mundo uma relação dialética, o homem produz a realidade, construindo e habitando um mundo com outros homens, tornando este mundo sua realidade definitiva e dominante, ao mesmo tempo em que o mundo atua sobre a natureza, transformando o homem, determinando a atividade humana, a consciência do ser e, até mesmo, o funcionamento do organismo biológico humano. Sendo assim, o processo de humanização do homem é variável em sentido sócio-cultural, ou seja, "a forma específica em que esta humanização se molda é determinada por essas formações sócio-culturais, sendo relativas às suas numerosas variações". Ou ainda, "o homem produz a realidade e com isso produz a si mesmo". Produto dessa dialética, a formação do homem deve ser "compreendida em relação com o contínuo desenvolvimento orgânico e com o processo social" no contexto particular em que ocorre. (Ibidem, p.72, 73 e 241)

\section{A QUESTÃO DA IDENTIDADE.}

No processo de interação homem/mundo interessa-nos a dialética que produz identidade. Segundo Berger e Luckmann (1973), identidade

- é um fenômeno que se cristaliza / cristalizável, importante da realidade subjetiva; e está em relação dialética com a sociedade;

- relação Dialética por ser formada por processos sociais determinados pela estrutura 
social e que, ao cristalizar-se, é mantida ou modelada pelas relações sociais; sendo que, tais identidades reagem sobre a estrutura social podendo modelá-la.

Retomando as idéias inicialmente apresentadas, as identidades se produzem pelas interações do organismo, da consciência individual e da estrutura social, das quais emergem as identidades individuais e os tipos de identidade ou identidades tipificadas. ${ }^{3}$

Assim como identidades particulares emergem no curso da história das sociedades, os tipos de identidades são engendrados por estruturas sociais históricas particulares. Podem ser observados na vida cotidiana, reconheciveis em casos individuais, verificáveis como tal (tipificadamente) ou refutados no nivel pré-teórico pelos homens comuns.

\section{A PESSOAÉ - EM IDENTIDADE, E EM IDENTIDADES TIPIFICADAS!}

As tipificações são importantes para a orientação e o comportamento dos indivíduos na vida cotidiana, sendo que os papéis são formas de ação tipificadas. Sua implicação para o individuo é a identificação da sua personalidade com o papel que desempenha - com o sentido objetivo atribuido socialmente a sua ação. Desempenhando vários papéis na vida social, todo um segmento da sua personalidade objetiva-se em termos de papéis a desempenhar. Este é "o verdadeiro eu social [embora] subjetivamente experimentado como distinto do eu em sua totalidade, chegando mesmo a defrontar-se com este" (Ibidem, p.102).

Temos, então, que a identidade tipificada - o enfermeiro, se constrói a partir da inserção na profissão e continuamente no cotidiano do trabalho, e é marcante na construção da identidade pessoal.

A construçăo de tipos de papéis é elemento necessário na institucionalização da conduta humana. A cada instituição são elencados diversos tipos de papéis a serem desempenhados pelos individuos que a constituem. Sendo assim, os papéis são formas de controle institucional. "As instituições incorporam-se à experiência do indivíduo por meio dos papéis" (Ibidem, p.103).

\section{TEORIAS SOBRE IDENTIDADE}

A identidade é considerada um fenômeno social, localizado em um mundo, e sua teorização deve fazer-se "no quadro das interpretações teóricas em que são localizadas" (Ibidem, p.230). Ou seja, a teorização sobre identidade deve ser sensivel ao contexto sócio-cultural, reconhecendo na situação social do individuo as definições da realidade admitidas como verdadeiras. A identidade, fenômeno socialmente definido, deve ser referida às definições sociais da realidade em geral.

De início, apresentamos a inter-relação dos conceitos de Cultura e Identidade dentre os diversos que se apresentam. No entanto, também não se pretende limitar esta construção de identidade a de habitus constituidos, numa linearidade entre cultura/habitus/idêntitas. No estudo sobre identidade, deverá ser necessário considerar ainda:

- que o homem não está dado definitivamente, ele se produz através da história, nas práticas que são o lócus de emergência de suas formas. E, aqui, pode-se focalizar, especialmente,

\footnotetext{
"Identidade individual "é um fenômeno que deriva da dialética entre um individuo e a sociedade"; e tipos de identidade "são produtos sociais tout court, elementos relativamente estáveis da realidade social objetiva" (Berger e Luckmann, 1973, p.230). Tout court: "loc. adv. - sem mais nada". (Corrêa, 1980, p.144).
} 
sobre o trabalho, como espaço de produção, objetivação e expressão humana; portanto, o trabalho é lócus importante de emergência das formas de identidade individual e tipificada;

- que a cultura - processo de produção do homem, é permeada pelo poder, que fornece uma importante perspectiva/delimitação do homem. O mesmo que dizer que a identidade é constituída sempre em uma certa relação (e relaçס̃es abrigam forças e embates) com o onde o homem está situado. Assim, a relação identidade e cultura é mediada, também, por uma concepção de poder.

Entre os diversos pontos de reflexão, cabe levantar se os hábitos constituídos (que engendram identidades/identidades tipificadas) podem se configurar como estratégia de poder diluída na cultura social. É necessário, também, estabelecer a relação poder e trabalho. Ou melhor, é preciso localizar o poder no trabalho.

O trabalho possui referência histórica e eixos estruturantes que se situam dentro e fora do próprio trabalho, incluindo elementos da ordem da própria construção do saber. Este, até certo ponto é especificável, objetivado em modos de intervenções e tecnologias e destinado a se concretizar em instituições reconhecidas para tal. Assim é que o trabalho, enquanto tipificador de papéis, no campo da saúde se delimita, em especial, através do saber, e do saber operacionalizado em condutas e técnicas ${ }^{4} \mathrm{O}$ estudo da relação saber-poder no trabalho pode contribuir para o entendimento da construção de Identidade, em especial, da Identidade Tipificada do trabalhador da Enfermagem.

Este e outros pontos remetem, ainda, ao reconhecimento de que a relação identidade e cultura precisa ser mediada por uma concepção de poder que não se limite ao campo do trabalho, como poder externo aplicável ao trabalhador e aos processos de produção, mas que incorpore o trabalho como ação humana essencial, também constituinte de uma identidade humana histórica e expressão da rede de elementos que participam desta construção, entre os quais o poder tem papel estruturante. Assim é que as transformações do mundo do trabalho fazem incidir mudanças tanto sobre os processos produtivos concretos em si, como sobre a relação subjetiva que o ser humano estabelece com e no seu trabalho.

\section{IDENTIDADE E TRABALHO}

Para Marx (1983), o fundamento de toda vida social é a produção material. Esta se dá pelo trabalho humano, que se distingue do trabalho instintivo/primitivo, sendo especificamente humano por sua Intencionalidade - segue, em seus passos e resultados, um projeto idealizado pelo homem. O trabalho é definido como o processo de intercámbio material entre o homem e a natureza no qual o homem atua, intencionalmente, sobre os elementos da natureza, transformando-os em valores de uso em atendimento às suas necessidades.

Um argumento primário desta relação trabalho identidade, pode ser buscado em Markus [19__, ao lembrar que a consciência humana é tanto social - determinada pelo movimento social enquanto processo histórico / natural e governada por leis independentes da vontade; quanto natural - ou cogniscível; e sensitiva - ou sensível. (Ibidem, p. 68-69).

A consciência humana - incluindo a consciência sensível, dispõe de dupla mediação: "sua relação com o objeto é mediatizada pela sociedade, pelo grau ao qual a consciência social já chegou, bem como pelo conhecimento, que deve ser apropriado (pelo menos em parte) pela

4 Voltando a Berger e Luckmann, op. cit., o desempenho de formas de ação tipificadas construtoras de identidades tipificadas, está vinculada ao conhecimento, estruturado por sua vez em termos de poder. 
consciência humana"; e "seu desenvolvimento individual é mediatizado pela atividade humana em sua forma principal e indivisa, ou seja, pela atividade de trabalho". Assim, a atividade de trabalho inclui as formas de consciência sensivel - aquela na qual parece haver uma relação bastante próxima e imediata entre a consciência e o objeto. (Ibidem, p. 63-64).

Sendo assim, a atividade do trabalho é um importante processo na construção da identidade do homem. ${ }^{5}$

Pela relação aqui ressaltada, natureza-homem-sociedade, interessa-nos a mediação, possivel pelo trabalho nesta relação. Neste aspecto cabe o reconhecimento básico "do duplo caráter do trabalho; como processo produtivo e formativo" (Ramos, 1995, p. 42). Ou seja, mesmo reconhecendo a impossibilidade de uma aplicação direta e linear dos conceitos marxianos, pelas próprias metamorfoses do mundo do trabalho, não se pode negar a importância deste pressuposto fundamental, uma vez que a partir dele situa-se o trabalho como elemento importante não apenas na macro-análise dos sistemas produtivos, revoluções tecnológicas e novas feições do trabalho e consumo de riquezas e produções sociais, mas, inclusive, dos limites e possibilidades deste trabalho veicular uma identidade de seus agentes ou de permitir relações deste ou daquele tipo entre produtor, produto e consumidor. Em outras palavras, se devemos evitar interpretações ortodoxas de Marx, devemos também evitar que o preconceito e os limites de análise nos impeçam de buscar um revigoramento do ceme politico desta visão, exatamente por possibilitar considerar este duplo caráter do trabalho; principalmente, considerá-lo como momento privilegiado de construção e realização humana. (Leo Maar, 1993)

Referindo-se ao modo como o trabalho, frente à fragmentação, desestabilização e outras tantas manifestações de sua versão mais atual, perdeu o espaço central na experiência subjetiva do homem, a partir da qual se configurava (o trabalhador) como sujeito privilegiado da história, diz Ramos (1995, p.43): "Afinal, se a centralidade subjetiva do trabalho evidenciou a marginalidade objetiva do sujeito, os sinais da ruptura desta centralidade podem ser os sinais de uma possibilidade histórica peculiar, a de resgate da esquecida face do trabalho enquanto processo formador do sujeito e, portanto, fator de desalienação e libertação deste sujeito através de seu próprio agir (dentro e fora do trabalho)."

\section{TRABALHO}

Marx (1991) nos diz que a produção humana é determinada socialmente, os individuos produzem em sociedade, ou melhor, em um determinado grau de desenvolvimento social, ou seja, a produção tem relação com as condiçöes sociais de produção.

Porém, a todos os graus de produção existem traços comuns - conjunto complexo de determinações, apreendidos pelo pensamento como gerais. São chamados condições gerais de toda a produção. São fatores abstratos os quais não explicam nenhum grau histórico efetivo da produção. ${ }^{6}$

Embora sendo produto de condições históricas especificas, Marx elaborou a abstração

${ }^{5}$ Para Almeida (1997, p. 24), o trabalho "é o modo como o homem produz e reproduz sua existência. Ao fazê-lo, estabelece relaçöes sociais e objetiva sua subjetividade. A concepção histórica, materialista e dialética procura demonstrar que [...] as circunstâncias fazem os homens, da mesma forma que os homens fazem as circunstâncias".

${ }^{6}$ Embora que *...até as categorias mais abstratas [...] apesar de sua validade para todas as épocas, são, contudo, na determinidade desta abstração, igualmente produto de condiçōes históricas, e não possuem plena validez senão para estas condiçōes e dentro dos limites destas" (Marx, 1991. p.20). Assim sendo, a categoria Trabalho é contextualizada na sociedade burguesa moderna. 
da categoria Trabalho pois considerava que, para compreender o concreto (sintese de múltiplas determinações - unidade da diversidade) era necessário reduzir a plenitude da representação a uma determinação abstrata. Assim, o Trabalho Humano Abstrato (Ramos, 1998) é uma determinação abstrata - uma substância social, universal/concreto/singular. Esta abstração contém os trabalhos concretos e particulares e através dela o concreto pode, pela via do pensamento, ser reproduzido. ${ }^{7}$

Esquematicamente e sucintamente, os elementos componentes do trabalho, tomado como Processo de Trabalho, segundo Marx (1983), são: Objeto, Instrumental ${ }^{8}$,Força de Trabalho e Produto.

Frente ao produto acabado, é indiferente a nós o trabalho que lhe imprimiu forma. $O$ produto do trabalho humano conduz a novo processo de trabalho, uma vez gerar novas necessidades que devem ser atendidas. As Necessidades Humanas, por sua vez, são sociais, uma vez produzidas no meio social e atendidas por processos sociais - em especial, pelo processo de trabalho.

\section{O trabalho em saúde}

A partir de inúmeros estudos, entre os quais podemos citar os de Gonçalves (1994), Almeida (1989), Leopardi, (1991), Ramos (1995, 1999), e Pires (1989, 1999), Leopardi et all (1999), podemos nos debruçar sobre diferentes focos e relevâncias sobre o processo de trabalho em saúde, tendo em comum o seu entendimento enquanto prática social articulada na estrutura de nossa sociedade capitalista. ${ }^{9}$

O processo de trabalho em saúde, segundo Pires (1999, p.28), tem como Finalidade a ação terapêutica de saúde; o Objeto - o individuo ou grupos de indivíduos com necessidades de saúde (afetadas ou não); o Instrumental - "os instrumentos e as condutas que representam o nivel técnico do conhecimento que é o saber de saúde [sem itálico no original]", ou seja, os saberes objetivados; e como produto final a própria prestação da assistência de saúde. ${ }^{10}$

\footnotetext{
7 Para melhor entender o que é esta determinação abstrata, devemos procurar em "O método da economia política", In Contribuição à crítica da economia politica, Marx (1977) - que nos traz, também, sua compreensão da realidade e do conhecimento da realidade; $\theta$ a dialética em seu método.

"O desenvolvimento da força de trabalho e as condições sociais em que o trabalho se realiza podem ser avaliados pelos meios de trabalho - que nos diz como, e com que meios o produto foi realizado. (Marx, 1983). Gonçalves (1994) redefine o conceito de Tecnologia para Organização Tecnológica do Trabalho e, ao abordar os instrumentos do trabalho como tecnologias, nos traz de forma ampliada a articulação: meios de produção/condições sociais de produção.

- Marx (1991, p.5) nos fala que a produção é sempre certo corpo social, que "exerce sua atividade numa totalidade maior ou menor de ramos da produção". O processo de trabalho em saúde segue, então, a lógica do modo de produção capitalista, faz parte do setor de serviços; tem características comuns (dentre as quais a divisão social e técnica do trabalho), assim como, especificas (entre elas, a prestaçäo do serviço de saúde pode assumir diversas formas; existem inúmeras categorizações das açöes e tipos de ações).

$10 \mathrm{O}$ processo de trabalho em saúde esta na esfera da produção năo material, aquela na qual o trabalho se consome e se completa no ato da sua realização: "o produto é indissociável do processo que o produz". (Pires, 1999, p.28).
} 


\section{INSTRUMENTOS TECNOLÓGICOS DO TRABALHO EM SAÚDE}

Gonçalves (1994, p.31) propôs-se ao estudo de tecnologias "enquanto forma variável e contraditoriamente adequada de organizar 'internamente' certas práticas referidas à saúde e à doença, ao mesmo tempo em que forma variável e contraditoriamente adequada de suportar a articulação dessas práticas na totalidade social histórica". Entendendo por forma variável que tecnologias são conjunto de saberes e suportes materiais do saber (instrumentos), e por "contraditoriamente adequada", que no processo produtivo as tecnologias expressam "a rede de relações sociais em que seus agentes articulam sua prática em uma totalidade social". Assim, as tecnologias ficam situadas numa realidade social que é dinâmica (e portanto histórica) e repleta de tensões e conflitos. (Ibidem, p.32).

O mesmo autor nos fala em Instrumentos Tecnológicos (entre os quais, o saber) como os componentes do processo de trabalho que intermediam e possibilitam a atuação do homem sobre o objeto de trabalho, modificando-Ihe sua natureza, presumindo que este saber pode ser apreendido ao se objetivar nas representações dos trabalhadores "e nas características do próprio processo de trabalho, no qual apresenta-se uma certa organização tecnológica.

Esta organização tecnológica inicia-se pelo modo como este saber possibilitará apreender o objeto de trabalho; o saber passa a produzir um certo objeto para o trabalho. Dentre os saberes, o saber médico definiu seu objeto e construiu o conhecimento sobre o objeto - 0 objeto foi concebido e, mais ainda, essa concepção foi fundamentada cientificamente estruturando-se sobre ciências positivas, das quais derivou a medicina experimental. E esse objeto, apreendido por essas ciências positivas e tornado Objeto do trabalho, passa a ser aceito como o Objeto verdadeiro e único. ${ }^{12}$ (Ibidem, p.63).

Sendo o saber uma tecnologia, é improdutiva a crítica do conhecimento "em si mesmo" (no seu âmbito exclusivo) uma vez que o conhecimento estende-se ao real onde encontra seu sentido, e onde devemos buscar suas conexões ao nivel interno "de uma certa prática [...] e ao mesmo tempo em função das articulações sociais que a determinam". (Ibidem, p.85)

\section{SÃO OS SABERES (DE ENFERMAGEM) PRODUTORES DE (SUA) IDENTIDADE?}

É necessário estabelecer a relação poder e trabalho. Ou melhor, é preciso localizar o poder no trabalho. O desempenho de formas de ação tipificadas - construtoras de identidades tipificadas, está vinculado ao conhecimento, estruturado por sua vez em termos de poder (Berger e Luckmann, 1973). O estudo do saber enquanto forma de poder no trabalho pode contribuir para o entendimento da construção de Identidade, em especial, da Identidade Tipificada do trabalhador da Enfermagem.

O saber não é autônomo por referência ao mundo real. Há de se buscar o sentido tecnológico do saber. E quanto a "qualquer aplicação tecnológica: a racionalidade que preside os processos de produção não se situa no plano do conhecimento ou da boa vontade, mas nas relaçð̌es sociais significativas [sem itálico no original] em que tais processos se inserem". (Gonçalves, 1994, p.90).

Por conseguinte, a análise da identificação da enfermagem - sua identidade tipificada,

"Gonçalves (1994, p.40) reconhece as dimensões subjetivas do processo de objetivação como expressivas do próprio processo de objetivação - "toma-se as representações dos agentes do trabalho como constitutivas do mesmo".

${ }^{12}$ Ainda segundo Gonçalves (1994, p.63), Outros objetos e saberes sobre o objeto de trabalho são desqualificados por "não verdadeiros" passando tal atributo do plano do conhecimento para a legitimação no processo de trabalho em saúde "na base da interferéncia estatal sobre os trabalhos dos agentes de cura, a qual se verificou generalizadamente no Ocidente capitalista". 
através de seus saberes somente pode ser tentada se entendermos o saber enquanto tecnologia. ${ }^{13}$

Nos estudos de enfermagem é comum tomá-la como profissão, ou seja, socialmente reconhecida e com um estatuto e pauta de ações legalizados no plano formal. Mas tomar uma profissão apenas em seu estatuto científico restringe o conhecimento da mesma, pois os critérios técnico-científicos de competência e os valores ético-profissionais nos dizem apenas dos padrões normativos e técnicos. Desvinculada do mundo do trabalho, a profissão é instalada acima e além das leis sociais e exaltada em termos de valores.

Considerar a enfermagem como trabalho é a possibilidade de apreendê-la em suas contradições, possibilidades, limites e articulações na dinâmica do real. Sair do contexto da profissão em direção ao contexto conflitante do trabalho é reconhecer que os trabalhos em saúde são complementares, e é procurar qual o espaço institucional e social de cada um. É reconhecer, enfim, que o trabalho em enfermagem é uma prática social e histórica. (Almeida e Rocha, 1997).

\section{A INTER-RELAÇÃO DOS CONCEITOS}

O TRABALHO do ser humano

- O trabalho, exclusivamente humano em sua natureza, é interação do homem nesse mundo, que pelo mesmo se manifesta; assim como forma que produz o homem - e o produz em identidade.

- O trabalho é espaço de produção e objetivação, assim como, é lócus de construção e expressão humana, porquanto instância nunca totalmente dominada pelos

- determinantes que sobre ele pesam.

\section{O trabalho EM ENFERMAGEM}

- Os suportes cientificamente estruturados do saber em enfermagem orientam o trabalho de enfermagem (embora contraditoriamente) e apresentam uma conexão importante com a identidade ${ }^{14}$ de seus trabalhadores. Os saberes (e o fazer em enfermagem) expressam e operam identidades (e também aqui de forma contraditória).

- Os saberes configuram-se na dinâmica do trabalho em saúde e enfermagem, ou seja, devem ser concebidos como articulados à práxis do trabalho, à qual vivamente se aplicam, $\mathrm{e}$ serem referidos aos nexos históricos dinâmicos (em suas múltiplas determinações, entre as quais, culturais) da produção de serviços de saúde em nossa sociedade.

Considerando, além disso, a inter-relação dos conceitos de identidade, cultura e trabalho, como bases para o entendimento do processo de construção de identidades do trabalhador contemporâneo, especificamente do profissional enfermeiro, têm-se finalmente que levar em conta:

- Uma compreensão do trabalho que remeta às particularidades do atual processo de produção dos serviços de saúde no Brasil, incorporando as mudanças que as modernas

13 Para Leopardi (1998), os instrumentos de trabalho são de fundamental importância na organização tecnológica do trabalho, são os meios necessários para sua realização. Sua escolha deve estar articulada ao processo como um todo coerente. Considera que os instrumentos próprios de cada profissão podem configurar sua identidade, uma vez que a finalidade e o objeto do trabalho são os mesmos para as profissões da saúde. Os instrumentos qualificam os agentes!

${ }_{14}$ Desejo dizer aqui tanto a identidade do ser individual (uma vez que, no trabalho o homem se produz), quanto a identidade tipificada do trabalhador da enfermagem (que se verifica nos seus papéis). 
transformações do mundo do trabalho fazem incidir tanto sobre os processos produtivos concretos em si, como sobre a relação subjetiva que o sujeito estabelece com seu trabalho.

- Que uma consequeência da utilização de tais conceitos será a necessidade de apreensão deste trabalho em sua referência histórica, em eixos estruturantes que se situam dentro e fora do próprio trabalho como realidade imediata, incluindo elementos da ordem da própria construção de um saber até certo ponto especificável, objetivado em modos de intervenções e tecnologias e destinado a se concretizar em instituições reconhecidas para tal, contudo, sem deixar de valorizar a ordem do cotidiano onde tais práticas se dão, como instância nunca totalmente dominada pelos determinantes que sobre ela pesam, já que locus de construção e expressão do sujeito.

- Que a articulação entre dimensões objetivas e subjetivas do processo de construção da identidade profissional do enfermeiro, exigência para um pensamento que vise se defrontar com a complexidade e dimensão dialética do tema, terá que buscar o enfrentamento do desafio teórico-metodológico, considerando os limites de possiveis abordagens, ora privilegiando um ou outro elemento do processo, ou mesmo pela reconhecida impossibilidade de referenciais que abarquem categorias analíticas de tal complexidade e dinamicidade.

ABSTRACT: The purpose of this article is to reflect on the interrelation of the concepts of culture, identity and work. It is believed that this reflection is a contribution to studies which aim at understanding the process of the identity construction of the nursing professional. It is based on the premise that identity is built day by day in the work environment, and, among other references, within a given culture.

KEYWORDS: culture, identity, work, nursing

RESUMEN: El articulo pretende reflexionar sobre la interrelación entre los conceptos cultura, identidad y trabajo, entendiéndola como una contribución a los estudios que buscan comprender los procesos de construcción de la identidad del enfermero. Parte del presupuesto de que dicha identidad se construye en lo cotidiano del trabajo y -entre otras referencias- en una dada cultura.

PALABRAS CLAVE: cultura, identidad, trabajo, enfermería

\section{BIBLIOGRAFIA CONSULTADA}

HARRIS, Marvin. Vacas, porcos, guerras e bruxas: os enigmas da cultura. Trad. De Irma Fioranti. Rio de Janeiro: Civilizaçăo Brasileira, 1978.

LARAIA, Roque de Barros. Cultura: um conceito antropológico. Rio de Janeiro: Jorge Sahar Ed., 1996, p. 21-65.

SAHLINS, Marshall D. A cultura e o meio ambiente: o estudo de ecologia cultural. In: Sol Tax (Org.). Panorama da antropologia. Rio de Janeiro: Fundo de Cultura, [196?]. p.100-110.

VIETLER, Renate Brigitte. Ecologia Cultural: uma antropologia da mudança. São Paulo: Ed. Ática, 1988

\section{REFERÊNCIAS BIBLIOGRÁFICAS}

ALMEIDA, M. C. P. ; ROCHA, J. S. Y. O saber de enfermagem e sua dimensão prática. 2. ed. São Paulo: Cortez, 1989.

ALMEIDA, M. C. P.; ROCHA, S. M. M. Considerações sobre a enfermagem enquanto trabalho. In: (Org.). O trabalho da enfermagem. Săo Paulo: Cortez, 1997. p.15-26. 
BERGER, Peter e LUCKMANN, T. A construção social da realidade: tratado de sociologia do conhecimento. Tradução por Floriano de Souza Fernandes. 2. Petrópolis: Vozes, 1973.

CORREA, Roberto Alvim. Dicionário escolar francês-português, português-francês. 6. ed. rev. e atual. Rio de Janeiro: FENAME, 1980. 939 p.

GONÇALVES, Ricardo B. M. Tecnologia e organização social das práticas de saúde. São Paulo: Hucitec/Abrasco, 1994.

LEO MAAR, Wolfgang. O fim da sociedade do trabalho ou a emancipação crítica do trabalho social. Conferência proferida no Seminário Internacional "Liberalismo e Socialismo: velhos e novos paradigmas, abril/1993, UNESP, Marília. (mimeogr.)

LEOPARDI, Maria Tereza. Método de assistência de enfermagem: análise da utilização do instrumento no processo de trabalho. Ribeirăo Preto, USP, 1991. 177 p. Tese (Doutorado em Enfermagem) Escola de Enfermagem de Ribeirão Preto, Universidade de São Paulo, 1991.

Instrumentos de trabalho na saúde: razão e objetividade. In: LEOPARDI, M. T. et al. Processo de trabalho em saúde: organização e subjetividade. Florianópolis: Papa-Livros, 1999.

LEOPARDI. M. T. et al. Processo de trabalho em saúde: organização e subjetividade. Florianópolis: Papa-Livros, 1999.

MÁRKUS, György. A teoria do conhecimento no jovem Marx. [S.I.]: [s.n.], [19_]

MARX, Karl. In: __ O capital. Livro 1. Tradução por Reginaldo Santana. Ed. Civilização Brasileira,

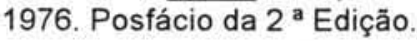

MARX, Karl. O capital. São Paulo: Abril Cultural, 1983. v.1, livro 1, O processo de trabalho e o processo de produzir mais valia. p. 149-163.

Contribuição à crítica da economia política. São Paulo: Martins Fontes, 1977. p. 218-220: o método da economia política.

Manuscritos econômicos filosóficos e outros textos escolhidos: seleção de textos de José Arthur Giannotti. Trad. José Carlos Bruni [et al.]. 5. ed. São Paulo: Nova Cultural, 1991. p.3-25.

Pequeno Dicionário Brasileiro da Língua Portuguesa. Ilustrado. 11. ed. 14. tiragem. Gamma

PIRES, Denise. Hegemonia médica na saúde e a enfermagem. Säo Paulo: Cortez, 1989

PIRES, Denise. A estrutura objetiva do trabalho em saúde. In: LEOPARDI, M. T. Processo de trabalho em saúde: organização e subjetividade. Florianópolis: Papa-Livros, 1999.

RAMOS, Flávia R. S. Para ler Marx. Cuiabá, 1998. Anotações de sala de aula, disciplina Processo de Trabalho e Conhecimento em Saúde. Mestrado em Saúde e Ambiente do ISC - UFMT.

Obra e manifesto: o desafio estético do trabalhador da saúde. Pelotas: UFPEL; Florianópolis: PEN/UFSC, 1995.

Quem produz e a quem o trabalho produz. In.: LEOPARDi. M. T., et al. Processo de trabalho em saúde: organizaçăo e subjetividade. Florianópolis: Papa-Livros, 1999.

Recebido em julho de 2000

Aprovado em dezembro de 2000 